\title{
Metallicity gradients in dwarf elliptical galaxies
}

\author{
Mina Koleva ${ }^{1,2,3}$, Philippe Prugniel ${ }^{3}$, Sven De Rijcke ${ }^{4}$ \\ and Werner W. Zeilinger ${ }^{5}$ \\ ${ }^{1}$ Instituto de Astrofísica de Canarias, La Laguna, E-38200 Tenerife, Spain \\ ${ }^{2}$ Departamento de Astrofísica, Universidad de La Laguna, E-38205 La Laguna, Tenerife, Spain \\ ${ }^{3}$ Université Lyon 1, Villeurbanne, F-69622, France; CRAL, \\ Observatoire de Lyon, St. Genis Laval, F-69561, France; CNRS, UMR 5574 \\ ${ }^{4}$ Sterrenkundig Observatorium, Ghent University, Krijgslaan 281, S9, B-9000 Ghent, Belgium \\ ${ }^{5}$ Institut für Astronomie, Universität Wien, Türkenschanzstraße 17, A-1180 Wien, Austria
}

\begin{abstract}
We discuss the relations between the metallicity gradients and the other characteristics of a set of dwarf elliptical galaxies in different environments $\dagger$. We suggest that dEs have typically metallicity gradients of $-0.30 \pm 0.25 \mathrm{dex} / \mathrm{r}_{e}$, unrelated to their mass. Dwarf elliptical galaxies with embedded disks, or dS0, may have flat metallicity gradients.
\end{abstract}

Keywords. galaxies: dwarf, galaxies: elliptical and lenticular, cD, galaxies: stellar content

\section{Introduction}

The dwarf elliptical galaxies (dEs) are likely the end-product of the evolution of lowmass galaxies, after the stripping of their gas and quenching of the star formation. The absence of a dazzling young population allows to see the older population and constrain its history and spatial distribution.

Some simulations of the formation of dwarf galaxies (e.g. Matteucci \& Tornambe 1987, Kawata \& Gibson 2003) predict that their shallow gravitational potentials prevent the building-up of a metallicity gradient. A consequence is the expected existence of a relation between the mass and the gradient. However, other SPH simulations on the dwarf formation predict gradients in dEs (e.g. Valcke et al. 2008).

In a recent paper (Koleva et al. 2009b), we found that in general the metallicity decreases from the center outwards, and the age does the opposite. Here we discuss how the metallicity gradients are related to other properties.

\section{Data and Analyses}

We obtained long-slit spectra with FORS1 and FORS2 on VLT for $16 \mathrm{dEs}$. Nine of them are situated in Fornax cluster and the other 7 in groups. Their luminosities range between -15.4 and -17.8 (absolute $B$ magnitude) and $B$ surface brightnesses between 21.4 and 23.3 and cover different sub-classes: (non)nucleated, with and without spiral structures, with or without gas. They are 'bright' dE galaxies, roughly comparable the NGC 205. The analysis was performed with the spectroscopic analysing package ULySS (Koleva et al. 2009a), doing a full spectrum fitting against stellar population models. The package is publicly availablef and its usage in this particular case is described in Koleva et al.

$\dagger$ Based on observations made with ESO telescopes at the La Silla Paranal Observatory under program ID076.B-0196

\section{$\ddagger$ http://ulyss.univ-lyon1.fr}



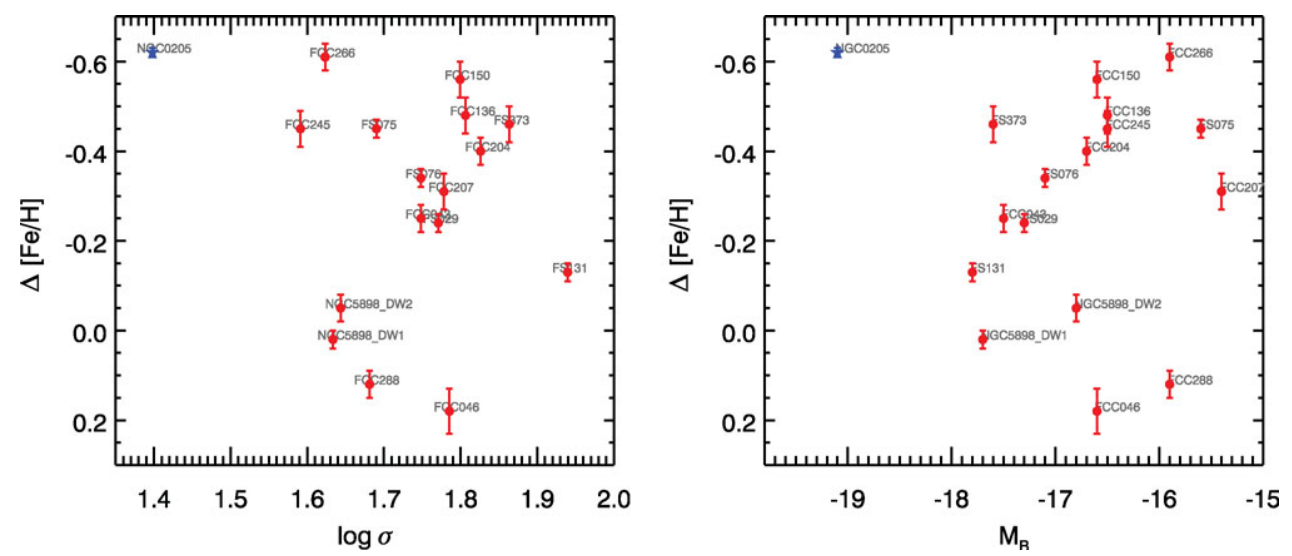

Figure 1. Metallicity gradients of our sample of 16 dwarf elliptical galaxies (red circles, Koleva et al., 2009b) and NGC 205 (a blue star) vs. their luminosity weighted velocity dispersion (left, De Rijcke et al. 2005) and absolute $B$-band magnitudes (right). The points are labeled with the corresponding galaxies' names.

(2009b). In order that our measurements are compatible with similar studies in the literature (e.g. Sánchez-Blázquez et al. 2006) we measured gradients by fitting a linear relation of the derived, SSP-equivalent metallicity vs. $\log _{10}\left(r / r_{e}\right)$.

\section{Results and Discussions}

We investigated if the mass of the galaxy (reflected in its physical velocity dispersion or its absolute magnitude) is correlated with the gradients (Fig.1).We did not find any correlation in our sample. We did not find differences between the group and cluster galaxies, nor between the nucleated and non-nucleated ones.

However, we do find a correlation with the suspected presence of an embeded disk, a strong apparent flatening or rotation. The 'flat' galaxies (with $\epsilon<0.3 \dagger$ ) have shallow gradients or even flat profiles. We may imagine that in spheroidal systems a small fraction of the metal is gradually deposited along the radius while in flat system, the metal escape along the short axis, and fountain back to enrich uniformly the disk. Another possibility is that the gradients in the spheroids are built from the gradual concentration of the star formation fed by gas of increasing metallicity (Valcke et al. 2008).

\section{References}

De Rijcke, S., Michielsen, D., Dejonghe, H., Zeilinger, W. W., \& Hau, G. K. T., 2005, A\&\&A, 438, 491

Koleva, M., Prugniel, Ph., Bouchard, A., \& Wu, Y. 2009, A\& A, 501, 1269

Koleva, M., De Rijcke, S., Prugniel, Ph., Zeilinger, W. W., \& Michielsen, D. 2009, MNRAS, 396, 2133

Matteucci, F. \& Tornambe, A., 1987, A\& $A, 185,51$

Kawata, D. \& Gibson, B. K., 2003, MNRAS, 340, 908

Paturel, G., et al., 2003, A\&BA, 412, 45

Sánchez-Blázquez, P., Gorgas, J., \& Cardiel, N., 2006, A\&A, 457, 823

Valcke, S., de Rijcke, S., \& Dejonghe, H., 2008, MNRAS, 389, 1111 